\title{
Stability of the Recovery of Missing Samples in Derivative Oversampling
}

\author{
Paola Brianzi \\ Dipartimento di Matematica, Università di Genova, \\ via Dodecaneso 35, 16146 Genova, Italia \\ brianzi@dima.unige.it \\ Vincenza Del Prete \\ Dipartimento di Matematica Università di Genova, \\ via Dodecaneso 35, 16146 Genova, Italia \\ delprete@dima.unige.it
}

\begin{abstract}
This paper deals with the problem of reconstructing a band-limited signal when a finite subset of its samples and of its derivative are missing. The technique used, due to P.J.S.G. Ferreira, is based on the use of a particular frame for band-limited functions and the relative oversampling formulas. We study the eigenvalues of the matrices arising in the procedure of recovering the lost samples, finding estimates of their eigenvalues and their dependence on the oversampling parameter and on the number of missing samples. When the missing samples are consecutive, the problem may become very ill-conditioned. We present a numerical procedure to overcome this difficulty, also in presence of noisy data, by using the Tikhonov regularization technique.
\end{abstract}

Key words and phrases: Frame, Riesz basis, oversampling, sampling formulas, band-limited functions.

2000 AMS Mathematics Subject Classification — 94A20, 41A15

\section{Introduction}

This paper deals with the problem of recovering missing samples of a band-limited function and of its derivative via frame reconstruction sampling formulas. A bandlimited signal is a function which belongs to the space $B_{\omega}$ of functions in $L^{2}(\mathbb{R})$ whose Fourier transforms have support in $[-\omega, \omega]$. Functions in this space can be expanded in terms of the orthonormal basis of translates of the sinc function. The coefficients of the expansion are the samples of the function at a uniform grid on $\mathbb{R}$, with "density" $\omega / \pi$ (Nyquist density). Such expansions, called sampling formulas, have been generalized by replacing the orthonormal basis with more general families, like Riesz bases and frames, formed by the translates of one or more functions (generators). Frames, unlike Riesz 
bases, are overcomplete and their redundancy provides a perfect tool for the recovery of missing data.

The recovery of missing samples of band-limited functions via oversampling has been first studied by R.J. Marks II in [10], [11]. In [4], P.J.S.G. Ferreira extended Marks' technique to generalized Kramer sampling, showing that, under suitable oversampling assumptions, any finite set of missing samples can be recovered from the others by solving a linear system $(I-S) X=B$, where the matrix $S$ is positive definite. In [5] the same author studied the eigenvalues of the matrix $S$ in dependence of the oversampling parameter and the number of missing samples; see also [6] for a related result. Successively, D.M.S. Santos and Ferreira considered the case of a two-channel derivative oversampling formula obtained by projecting the generators of a Riesz basis of the space $B_{\omega}$ and their duals into the space $B_{\omega_{a}}$ with $\omega_{a}<\omega$ [12], see also [13]. With this technique, they obtain a pair of dual frames, although the dual frame is not the canonical one. In their paper, the authors show that a finite number of missing samples either of the function or of its derivative can be recovered, solving in each case a non singular linear system similar to the one-channel case. In [13], the authors consider also the case when samples of both the function and the derivative are simultaneously missing. The technique of Santos and Ferreira has been applied to more general two-channels by J. M. Kim and K. H. Kwon [8], who gave sufficient conditions for the recovery of missing samples, from a single channel and from both channels. However, as the authors observe, these conditions do not apply to the derivative oversampling formula studied in $[12]$.

Successively, in [3], the author gave new derivative oversampling formulas of any order, where the dual generator is the canonical one and in [2] found an expression of the dual in the two-channel case. The use of the canonical dual allowed the author to prove in [2] that simultaneously missing samples of the function and of the derivative can be recovered. However, the generators of the canonical duals are much less explicit than the non-canonical dual used by Santos and Ferreira, see [2, (5.2) p. 178] and (2.10). In this paper we study the problem of the recovery of missing samples of the function and of its derivative in Ferreira's two-channel derivative formula. The technique we use, first proposed in [12], extends in a natural way the one for a single channel in [5] and consists in solving a system $(I-S) X=C$, where the matrix $S$ (see (3.8)) is a block matrix depending on the dual generators and on the position of the missing samples, and the unknowns $X$ are the missing samples of the function and its derivative. To the best of our knowledge, no results are known about the possibility of solving this system, i.e. of recovering simultaneous missing samples of the function and of its derivative.

We obtain estimates of the minimum and maximum eigenvalues of the block submatrices of the matrix $S$, show that in some cases the matrix reduces to a lower triangular matrix and provide several numerical experiments on the dependence of the eigenvalues on the parameters of the problem, i.e. the oversampling parameter $r$, the number of missing samples and their distance. Moreover, we experiment the recovery of missing samples and the reconstruction of a signal, paying particular attention to the computational aspects and to the ill-conditioned problem of contiguous missing samples, also with noisy data. We solve this problem by using the Tikhonov regularization, a technique typical of the treatment of inverse problems that consists in considering a family of approximate solutions $X_{\lambda}$ depending on a positive parameter $\lambda$, called the regularization parameter. When the data are noise-free, the solution $X_{\lambda}$ converges to the exact solution as the regularization parameter tends to zero. In the case of noisy data, one can obtain an 
optimal approximation of the exact solution for a positive value of the regularization parameter $\lambda([1]$, Section 5).

The paper is organized as follows. In Section 2 we establish notations and collect some results to be used later. In Section 3 we describe the technique for the recovery of the missing samples. Section 4 is dedicated to the study of the eigenvalues of the coefficient matrices in the system, mostly when the missing samples are equidistant. In this case we find estimates of the minimum and maximum eigenvalues of the diagonal submatrices $S_{11}$ and $S_{22}$ of $S$ (see (3.8)). Moreover, we find that, in some cases, the matrix $S$ is lower triangular and that half of its eigenvalues are equal to $2 r-r^{2}$, the other half are equal to $r^{2}$, where $r$ is the oversampling parameter. This extends a result in [5] to two channels. We also present some numerical experiments supporting the theory. Finally, in Section 5, we analyze the case of contiguous missing samples both for one and two channels, when the problem may become very ill-conditioned; we present a numerical procedure to solve it, also in the presence of noisy data.

\section{Preliminaries}

In this section we collect some results on frames to be used later. We begin by introducing some notation. The Fourier transform of a function $f$ in $L^{1}(\mathbb{R})$ is

$$
\mathcal{F} f(\xi)=\hat{f}(\xi)=\frac{1}{\sqrt{2 \pi}} \int f(t) e^{-i t \xi} d t .
$$

In this paper vectors in $\mathbb{C}^{N}$ are to be considered as column-vectors. However, to save space, we shall write $x=\left(x_{1}, x_{2}, \ldots, x_{N}\right)$ to denote the column-vector whose components are $x_{1}, \ldots, x_{N}$. Let $t_{o}$ be a positive number; we shall say that a closed subspace $H$ of $L^{2}(\mathbb{R})$ is $t_{o}$-shift-invariant if $H$ is invariant under translation by $t_{o}$. Given a subset $\Phi=\left\{\varphi_{j}: j=1,2\right\}$ of $H$, we denote by $E_{\Phi, t_{o}}$ the set

$$
E_{\Phi, t_{o}}=\left\{\tau_{n t_{o}} \varphi_{j}: n \in \mathbb{Z}, j=1,2\right\},
$$

where $\tau_{a} f(x)=f(x+a)$. The family $E_{\Phi, t_{o}}$ is a frame for $H$ if the operator $T_{\Phi, t_{o}}$ : $\ell^{2}\left(\mathbb{Z} ; \mathbb{C}^{2}\right) \rightarrow H$ defined by $T_{\Phi, t_{o}} c=\sum_{j=1}^{2} \sum_{n \in \mathbb{Z}} c_{j}(n) \tau_{n t_{o}} \varphi_{j}$ is continuous and surjective. It is well known that this happens if and only if there exist two constants $0<A \leq B$ such that

$$
A\|f\|^{2} \leq \sum_{j=1}^{2} \sum_{n \in \mathbb{Z}}\left|\left\langle f, \tau_{n t_{o}} \varphi_{j}\right\rangle\right|^{2} \leq B\|f\|^{2} \quad \forall f \in H .
$$

The constants $A$ and $B$ are called frame bounds. Denote by $T_{\Phi, t_{o}}^{*}: H \rightarrow \ell^{2}\left(\mathbb{Z} ; \mathbb{C}^{2}\right)$ the adjoint of $T_{\Phi, t_{o}}$, defined by $\left(T_{\Phi, t_{o}}^{*} f\right)_{j}(n)=\left\langle f, \tau_{n t_{o}} \varphi_{j}\right\rangle, n \in \mathbb{Z}, j=1,2$. The operator $T_{\Phi, t_{o}} T_{\Phi, t_{o}}^{*}: H \rightarrow H$ is called the frame operator. Denote by $\Phi^{*}$ the family $\left\{\varphi_{j}^{*}: j=\right.$ $1,2\}$, where

$$
\varphi_{j}^{*}=\left(T_{\Phi, t_{o}} T_{\Phi, t_{o}}^{*}\right)^{-1} \varphi_{j} \quad j=1,2 .
$$

If $E_{\Phi, t_{o}}$ is a frame for $H$, then $E_{\Phi^{*}, t_{o}}$ is also a frame of $H$, called the canonical dual frame, and $T_{\Phi, t_{o}} T_{\Phi^{*}, t_{o}}^{*}=T_{\Phi^{*}, t_{o}} T_{\Phi, t_{o}}^{*}=I$; explicitly

$$
f=\sum_{j=1}^{2} \sum_{n \in \mathbb{Z}}\left\langle f, \tau_{n t_{o}} \varphi_{j}^{*}\right\rangle \tau_{n t_{o}} \varphi_{j}=\sum_{j=1}^{2} \sum_{n \in \mathbb{Z}}\left\langle f, \tau_{n t_{o}} \varphi_{j}\right\rangle \tau_{n t_{o}} \varphi_{j}^{*} \quad \forall f \in H .
$$


The elements of $\Phi$ are called generators of the frame and the elements of $\Phi^{*}$ canonical dual generators. Given a frame $E_{\Phi, t_{o}}$ of a Hilbert space $H$, the expansion of an element in terms of translates of the generators is not unique; a frame $\left\{\tau_{k t_{o}} \varphi_{1}^{d}, \tau_{k t_{o}} \varphi_{2}^{d}: k \in \mathbb{Z}\right\}$ such that

$$
f=\sum_{j=1}^{2} \sum_{n \in \mathbb{Z}}\left\langle f, \tau_{n t_{o}} \varphi_{j}^{d}\right\rangle \tau_{n t_{o}} \varphi_{j} \quad \forall f \in H
$$

is called a dual frame for $E_{\Phi, t_{o}}$. We remind that the coefficients $\left\{\left\langle f, \tau_{n t_{o}} \varphi_{j}^{*}\right\rangle, n \in \mathbb{Z}, j=\right.$ $1,2\}$ with respect to the canonical dual frame have the minimal $\ell^{2}$ norm among all the sequences that represent an element $f$ in terms of a given frame $E_{\Phi, t_{o}}$. If the family $E_{\Phi, t_{o}}$ is a frame for $H$ and the operator $T_{\Phi, t_{o}}$ is injective, then $E_{\Phi, t_{o}}$ is called a Riesz basis.

Let $E_{\Phi, t_{o}}$ be a frame of a $t_{o}$-shift-invariant Hilbert space $H$ with the canonical dual generators $\Phi^{*}$. Denote by $P$ the orthogonal projection on a $t_{o^{-}}$-shift-invariant subspace $V$ of $H$, then $E_{P \Phi, t_{o}}$ and $E_{P \Phi^{*}, t_{o}}$ are families of dual frames for $V$, so that

$$
f=\sum_{j=1}^{2} \sum_{n \in \mathbb{Z}}\left\langle f, \tau_{n t_{o}} P \varphi_{j}^{*}\right\rangle \tau_{n t_{o}} P \varphi_{j}=\sum_{j=1}^{2} \sum_{n \in \mathbb{Z}}\left\langle f, \tau_{n t_{o}} P \varphi_{j}\right\rangle \tau_{n t_{o}} P \varphi_{j}^{*} \quad \forall f \in V .
$$

Note that $E_{P \Phi^{*}, t_{o}}$ is not necessarily the canonical dual.

In this paper we study families $E_{\Phi, t_{o}}, \Phi=\left\{\varphi_{1}, \varphi_{2}\right\}$ where $\varphi_{1}$ and $\varphi_{2}$ have Fourier transform $\widehat{\varphi_{1}}(\xi)=\chi_{[-\omega, \omega]}(\xi)$ and $\widehat{\varphi_{2}}(\xi)=i \xi \chi_{[-\omega, \omega]}(\xi)$ and $t_{o}$ and $\omega$ are such that $\omega \leq \frac{2 \pi}{t_{o}} \leq 2 \omega$. The interest of these families in applications resides in their connection with derivative sampling formulas for the space $B_{\omega}$. To simplify notation, throughout the paper we shall set

$$
h=\frac{2 \pi}{t_{o}} .
$$

The family $E_{\Psi, t_{o}}, \Psi=\left\{\psi_{1}, \psi_{2}\right\}$ defined by

$$
\widehat{\psi_{1}}(\xi)=\chi_{[-h, h]}(\xi) \quad \widehat{\psi_{2}}(\xi)=i \xi \chi_{[-h, h]}(\xi)
$$

is a Riesz basis for the space $B_{h}$ (see [7, p.135]). The Fourier transforms of the dual generators are

$$
\widehat{\psi_{1}^{*}}(\xi)=\frac{1}{h}\left(1-\frac{|\xi|}{h}\right) \chi_{[-h, h]}(\xi) \quad \widehat{\psi_{2}^{*}}(\xi)=\frac{i}{h^{2}} \operatorname{sign}(\xi) \chi_{[-h, h]}(\xi),
$$

hence the dual generators are

$$
\psi_{1}^{*}(x)=\frac{1}{\sqrt{2 \pi}} \operatorname{sinc}^{2}\left(\frac{h x}{2}\right) \quad \psi_{2}^{*}(x)=-\frac{1}{\sqrt{2 \pi}} x \operatorname{sinc}^{2}\left(\frac{h x}{2}\right),
$$

where $\operatorname{sinc}(x)=\sin (x) / x$. For any $f \in B_{h}$ the coefficients of the expansion in (2.2) are

$$
\left\langle f, \tau_{-n t_{o}} \psi_{1}\right\rangle=\sqrt{2 \pi} f\left(n t_{o}\right) \quad\left\langle f, \tau_{-n t_{o}} \psi_{2}\right\rangle=-\sqrt{2 \pi} f^{\prime}\left(n t_{o}\right) \quad \forall n \in \mathbb{Z} .
$$

Thus the expansion formula (2.2) becomes

$$
f(x)=\sum_{n}\left(f\left(n t_{o}\right) \operatorname{sinc}^{2} \frac{h}{2}\left(x-n t_{o}\right)+f^{\prime}\left(k t_{o}\right)\left(x-n t_{o}\right) \operatorname{sinc}^{2} \frac{h}{2}\left(x-n t_{o}\right)\right)
$$


for any $f \in B_{h}$. Note that the convergence is uniform. This formula is called a first order derivative sampling formula. The sampling frequency for an $N$-channel formula is $\omega /(N \pi)$ and is called Nyquist frequency. By using a frame instead of a Riesz basis, in [12] the authors obtained a two-channel derivative formula where the sampling frequency in each channel is greater than the Nyquist frequency $\omega /(2 \pi)$ (first order derivative oversampling formula). The frame is obtained by projecting on $B_{\omega}$ the Riesz basis $E_{\Psi, t_{o}}$ for $B_{h}$, where $h=2 \pi / t_{o}$ and $\omega<h$. We denote by $r$ the ratio

$$
r=\frac{\omega}{h}=\frac{\omega t_{o}}{2 \pi} .
$$

Since the projection commutes with the translations, to project the family $E_{\Psi}$ and the dual family, it is sufficient to project on $B_{\omega}$ the generators and the dual generators. Thus, by (2.4), we obtain that the generators of the frame $E_{\Phi, t_{o}}$ are defined by

$$
\widehat{\varphi_{1}}(\xi)=\chi_{[-\omega, \omega]}(\xi) \quad \widehat{\varphi_{2}}(\xi)=i \xi \chi_{[-\omega, \omega]}(\xi)
$$

and by (2.5) we obtain the Fourier transform of the dual generators

$$
\widehat{\varphi_{1}}(\xi)=\frac{r}{\omega}\left(1-\frac{r}{\omega}|\xi|\right) \chi_{[-\omega, \omega]}(\xi) \quad \widehat{\widetilde{\varphi}_{2}}(\xi)=i \frac{r^{2}}{\omega^{2}} \operatorname{sign}(\xi) \chi_{[-\omega, \omega]}(\xi) .
$$

A simple calculation shows that

$$
\begin{aligned}
& \widetilde{\varphi}_{1}(x)=\frac{1}{\sqrt{2 \pi}}\left[2 r(1-r) \operatorname{sinc}(\omega x)+r^{2} \operatorname{sinc}^{2}\left(\frac{\omega x}{2}\right)\right] \\
& \widetilde{\varphi}_{2}(x)=-\frac{1}{\sqrt{2 \pi}} x r^{2} \operatorname{sinc}^{2}\left(\frac{\omega x}{2}\right) .
\end{aligned}
$$

Thus we obtain the following derivative oversampling formula

$$
f=\sqrt{2 \pi} \sum_{k \in Z}\left(f\left(k t_{o}\right) \tau_{k t_{o}} \widetilde{\varphi}_{1}-f^{\prime}\left(k t_{o}\right) \tau_{k t_{o}} \widetilde{\varphi}_{2}\right) \quad \forall f \in B_{\omega} .
$$

We observe that $1 / r=2 \pi /\left(\omega t_{o}\right)$ is the ratio between the sampling frequency and the Nyquist frequency and that $r \in(0,1)$. We shall be mainly interested to the case $1 / 2<$ $r<1$, since for $0<r \leq 1 / 2$ it is possible to use one channel separately for the function and for its derivative [12], [13]. Note that if $r$ is close to 1 , the frame is close to a Riesz basis, while if $r$ is small, the frame is very redundant.

\section{$3 \quad$ The system for the recovery of missing samples}

In this section we briefly describe the method for the recovery of a finite number of missing samples via two-channel derivative oversampling. We may rewrite equation $(2.11)$ as

$$
f(x)=\sqrt{2 \pi} \sum_{i=1}^{2} \sum_{k \in Z}(-1)^{i-1} f^{(i-1)}\left(k t_{o}\right) \widetilde{\varphi}_{i}\left(x-k t_{o}\right)
$$


and computing the derivative of both sides, gives

$$
f^{\prime}(x)=\sqrt{2 \pi} \sum_{i=1}^{2} \sum_{k \in Z}(-1)^{i-1} f^{(i-1)}\left(k t_{o}\right) \widetilde{\varphi}_{i}^{\prime}\left(x-k t_{o}\right) .
$$

Here

$$
\begin{aligned}
& \widetilde{\varphi}_{1}^{\prime}(x)=\frac{2 r}{\sqrt{2 \pi}} \frac{1}{x}[(1-r)(\cos (\omega x)-\operatorname{sinc}(\omega x))+ \\
& \left.+r \operatorname{sinc}\left(\frac{\omega}{2} x\right)\left(\cos \left(\frac{\omega x}{2}\right)-\operatorname{sinc}\left(\frac{\omega x}{2}\right)\right)\right] \\
& \left.\widetilde{\varphi}_{2}^{\prime}(x)=-\frac{1}{\sqrt{2 \pi}} r^{2} \operatorname{sinc}\left(\frac{\omega x}{2}\right)\left[2 \cos \left(\frac{\omega x}{2}\right)-\operatorname{sinc}\left(\frac{\omega x}{2}\right)\right)\right] .
\end{aligned}
$$

Observe that $\widetilde{\varphi}_{1}^{\prime}(0)=0$.

Let $\mathcal{U}=\left\{l_{1}, l_{2} \ldots, l_{N}\right\} \subset \mathbb{Z}$ and let $\left\{f\left(l_{j} t_{o}\right), f^{\prime}\left(l_{j} t_{o}\right), 1 \leq j \leq N\right\}$ be the corresponding set of missing samples. By evaluating equations (3.1) and (3.2) in $\ell_{k} t_{o}$ and separating the unknown samples from the known ones, we obtain

$$
\begin{gathered}
f\left(l_{k} t_{o}\right)-\sqrt{2 \pi} \sum_{i=1}^{2} \sum_{j=1}^{N}(-1)^{i-1} f^{(i-1)}\left(l_{j} t_{o}\right) \widetilde{\varphi}_{i}\left(\left(l_{k}-l_{j}\right) t_{o}\right)=c_{k} \\
f^{\prime}\left(l_{k} t_{o}\right)-\sqrt{2 \pi} \sum_{i=1}^{2} \sum_{j=1}^{N}(-1)^{i-1} f^{(i-1)}\left(l_{j} t_{o}\right) \widetilde{\varphi}_{i}^{\prime}\left(\left(l_{k}-l_{j}\right) t_{o}\right)=c_{k+N}
\end{gathered}
$$

$1 \leq k \leq N$, where

$$
\begin{aligned}
c_{k} & =\sqrt{2 \pi} \sum_{i=1}^{2} \sum_{n \in \mathbb{Z} \backslash \mathcal{U}}(-1)^{i-1} f^{(i-1)}\left(n t_{o}\right) \tilde{\varphi}_{i}\left(l_{k} t_{o}-n t_{o}\right) \\
c_{k+N} & =\sqrt{2 \pi} \sum_{i=1}^{2} \sum_{n \in \mathbb{Z} \backslash \mathcal{U}}(-1)^{i-1} f^{(i-1)}\left(n t_{o}\right) \widetilde{\varphi}_{i}{ }^{\prime}\left(l_{k} t_{o}-n t_{o}\right) .
\end{aligned}
$$

Equations (3.5) form a system of $2 N$ equations in the $2 N$ unknowns

$$
f\left(l_{1} t_{o}\right), \ldots, f\left(l_{N} t_{o}\right), f^{\prime}\left(l_{1} t_{o}\right), \ldots, f^{\prime}\left(l_{N} t_{o}\right),
$$

which can be written in matrix form: denote by $S=S(\mathcal{U}, r)$ the real matrix

$$
S=\left[\begin{array}{ll}
S_{11} & S_{12} \\
S_{21} & S_{22}
\end{array}\right],
$$

where $S_{11}, S_{12}, S_{21}, S_{22}$ are the submatrices whose entries are

$$
\begin{array}{ll}
S_{11}(k, j)=\sqrt{2 \pi} \tilde{\varphi_{1}}\left(\left(l_{k}-l_{j}\right) t_{o}\right) & S_{12}(k, j)=-\sqrt{2 \pi} \tilde{\varphi_{2}}\left(\left(l_{k}-l_{j}\right) t_{o}\right) \\
S_{21}(k, j)=\sqrt{2 \pi}{\tilde{\varphi_{1}}}^{\prime}\left(\left(l_{k}-l_{j}\right) t_{o}\right) & S_{22}(k, j)=-\sqrt{2 \pi}{\tilde{\varphi_{2}}}^{\prime}\left(\left(l_{k}-l_{j}\right) t_{o}\right)
\end{array}
$$


$k, j=1, \ldots, N$. Then the system (3.5) may be written

$$
(I-S) Z=C,
$$

where $I$ is the $2 N \times 2 N$ identity matrix, $C=\left(c_{k}\right)_{k=1}^{2 N}$ is defined by (3.6) and $Z=$ $\left(X_{1}, X_{2}, \ldots X_{N}, Y_{1}, Y_{2}, \ldots Y_{N}\right)$. The unknowns $X_{k}$ are the missing samples of the function and the $Y_{k}$ are the missing samples of the derivative.

Notice that, by (2.10), (3.3) and (3.4), if $r=1$, then $S$ is the identity matrix. The four submatrices are real, $S_{11}$ and $S_{22}$ are symmetric, while $S_{12}$ and $S_{21}$ are antisymmetric. Moreover, if the distance between any two consecutive $l_{k}$ 's is a constant, then the four submatrices are Toeplitz, but $S$ is not Toeplitz. Note that for $r \neq 1$ the matrix $S$ is neither symmetric nor antisymmetric.

Suppose that in (3.7) only the values $\left\{X_{i}=f\left(l_{i} t_{o}\right): i=1, \ldots, N\right\}$ are missing, while all the sample values of $f^{\prime}$ are known. By considering only the first $N$ equations of the system (3.10) and by separating the known from the unknown samples, we obtain

$$
\left(I-S_{11}\right) X=C_{1}+S_{12} Y,
$$

where $I$ is the $N \times N$ identity matrix, $X=\left(X_{k}\right)_{k=1}^{N}, Y=\left(f^{\prime}\left(l_{k} t_{o}\right)\right)_{k=1}^{N}$ and $C_{1}=$ $\left(c_{k}\right)_{k=1}^{N}$. This equation may be rewritten as

$$
\left(I-S_{11}\right) X=B_{1}
$$

where $B_{1}=C_{1}+S_{12} Y$. Similarly, if in (3.7) only the values $\left\{Y_{i}=f^{\prime}\left(l_{i} t_{o}\right), i=1, \ldots, N\right\}$ are missing, and the samples of $f$ are known, one solves the system

$$
\left(I-S_{22}\right) Y=B_{2}
$$

in the unknowns $Y_{i}, i=1, \ldots, N$, where $C_{2}=\left(c_{k}\right)_{k=1+N}^{2 N}$ and $B_{2}=C_{2}+S_{21} X$.

If $r \in(0,1)$, then the eigenvalues of the symmetric matrices $S_{11}$ and $S_{22}$ are in the interval $(0,1)[12]$. This implies that the recovery of a finite number of missing samples of the function is possible, if all the samples of the derivative are known and, vice-versa, samples of the derivative can be recovered, if all the samples of the function are known. In the next section we shall find bounds for the minimum and maximum eigenvalues of the matrices $S_{11}$ and $S_{22}$.

To the best of our knowledge, no results are known about the possibility of solving system (3.10), i.e. of recovering simultaneous missing samples of the function and of its derivative. Notice that the system is solvable if and only if 1 is not an eigenvalue of the matrix $S$. On the basis of numerical evidence, we conjecture that all the eigenvalues of $S$ are real and that they lie in the interval $(0,1)$ for all $r \in(0,1)$. In Section 4 we present several numerical experiments supporting our conjecture.

\section{The stability of the matrices.}

This section is dedicated to the study of the eigenvalues of the matrices $S, S_{11}$ and $S_{22}$ in the two-channel system (3.10). First, we briefly summarize the one-channel case and recall some stability results obtained in [5]. The one-channel formula is

$$
f(x)=\frac{\omega t_{o}}{\pi} \sum_{n \in \mathbb{Z}} f\left(n t_{o}\right) \frac{\sin \omega\left(x-n t_{o}\right)}{\omega\left(x-n t_{o}\right)} \quad \forall f \in B_{\omega},
$$


where $t_{o}<\frac{\pi}{\omega}$. In this case the oversampling parameter is $r=\omega t_{o} / \pi$. Let $U=$ $\left\{l_{1}, l_{2}, \ldots, l_{N}\right\}$ be a set of integers and

$$
\left\{f\left(l_{k} t_{o}\right): k=1, \ldots, N\right\}
$$

be the set of missing samples; then the system to be solved is

$$
(I-R) X=B
$$

where $I$ is the identity $N \times N$ matrix,

$$
\begin{gathered}
R(j, k)=r \operatorname{sinc}\left(\pi r\left(l_{j}-l_{k}\right)\right), \quad j, k=1, \ldots, N, \\
X=\left\{f\left(l_{k} t_{o}\right), k=1, \ldots, N\right\}, B=\left(b_{1}, b_{2}, \ldots, b_{N}\right), \text { and } \\
b_{j}=r \sum_{k \notin U} f\left(k t_{o}\right) \operatorname{sinc}\left(\pi r\left(l_{j}-l_{k}\right)\right), \quad j=1, \ldots, N .
\end{gathered}
$$

The matrix $R$ is symmetric and positive definite. In [5], the author shows that all its eigenvalues are in $(0,1)$ and observes that there are two situations that lead to a illconditioned problem: when $r$ is close to 1 and when the integers $l_{k}$ are contiguous. In the first case, the frame is close to a Riesz basis, when the recovery is impossible and $R$ becomes the identity matrix. In the second case, the maximum eigenvalue of the matrix $S$ grows rapidly with $N$; moreover it can be close to 1 also for small values of $N$ (see Figure 5 in [5]). In both cases, the spectral condition number of the matrix $(I-R)$, which controls the propagation of errors on the data, becomes very large.

Next, we investigate the eigenvalues of the matrix $S$ in the two channel case (see (3.8)) and of its submatrices $S_{11}$ and $S_{22}$. Since the latter are real symmetric matrices, their eigenvalues give their condition number. We shall denote by $\lfloor a\rfloor$ the maximum integer less than or equal to $a$. Given an $N \times N$ matrix, we shall denote with $\lambda_{j}(A), j=1, \ldots, N$ its eigenvalues and by $\lambda_{\min }(A)$ and $\lambda_{\max }(A)$ its minimum and maximum eigenvalue. Let $\mathcal{U}=\left\{l_{1}, l_{2} \ldots, l_{N}\right\} \subset \mathbb{Z}$ and let $\left\{f\left(l_{j} t_{o}\right), f^{\prime}\left(l_{j} t_{o}\right) 1 \leq j \leq N\right\}$ a set of missing samples. Then

$$
\begin{aligned}
& \lambda_{\min }\left(S_{11}\right)<2 r-r^{2}<\lambda_{\max }\left(S_{11}\right) \\
& \lambda_{\min }\left(S_{22}\right)<r^{2}<\lambda_{\max }\left(S_{22}\right),
\end{aligned}
$$

where $r$ is the oversampling parameter (2.7). Indeed, by (3.9) and (2.10), the trace of $S_{11}$ is $N\left(2 r-r^{2}\right)$; by (3.9) and (3.4), the trace of $S_{22}$ is $N r^{2}$. Moreover, since $S$ is a real matrix, its trace is $N\left(2 r-r^{2}\right)+N r^{2}=\sum_{i=1}^{2 N} \operatorname{Re}\left(\lambda_{i}(S)\right)$; hence

$$
\operatorname{Re}\left(\lambda_{\min }(S)\right)<r<\operatorname{Re}\left(\lambda_{\max }(S)\right) .
$$

We observe that, if the oversampling parameter $r$ tends to 1, i.e. the frame tends to a Riesz basis, then $S$ tends to the identity matrix.

Following Ferreira, we shall now consider the case in which the set that locates the positions of the missing samples is $\mathcal{U}=\left\{m i_{1}, m i_{2}, \ldots, m i_{N}\right\}$, where $m$ is an integer and $\mathcal{I}=\left\{i_{1}, i_{2}, \ldots i_{N}\right\}$ is a set of integers; in the following we shall denote such sets by $m \mathcal{I}$. The interest for studying these cases lies in the technique of interleaving the samples of a signal, prior to their transmission or archival; the advantage of this 
procedure is that the transmitted (or stored) information becomes less sensitive to the burst errors that typically affect contiguous set of samples [5]. We shall investigate how the stability of the method depends on the interleaving factor $m$. In Proposition 4.1 and Theorem 4.2 below we find estimates for the eigenvalues of the matrices $S_{11}$ and $S_{22}$, thus generalizing a result of Ferreira for one channel [4, Theorem 1].

First, we consider the case of integer $m r$. The following proposition, which extends to two channels a result in [5], shows that in this case the matrix $S$ is lower triangular and that $N$ eigenvalues are equal to $2 r-r^{2}$ and $N$ are equal to $r^{2}$. Hence, if $r$ is rational $r=p / q$, one can take $m$ to be a multiple of $q$ and obtain a lower-triangular matrix $S$.

Proposition 4.1. Let $m$ be a positive integer; suppose $\mathcal{U}=\left\{m i_{1}, m i_{2}, \cdots, m i_{N}\right\}$, where $i_{j}, j=1, \ldots, N$, are integers and let $r$ be a real number in $(0,1)$. If $m r$ is an integer, then

$$
S_{11}=\left(2 r-r^{2}\right) I \quad S_{22}=r^{2} I \quad S_{12}=0 .
$$

Moreover the entries of the matrix $S_{21}$ are

$$
S_{21}(k, j)= \begin{cases}(1-r) \omega /\left(m \pi\left(i_{k}-i_{j}\right)\right) & \text { if } k \neq j \\ 0 & \text { if } k=j .\end{cases}
$$

Proof. Since $t_{o}=2 \pi r / \omega$, by (3.9) with $\ell_{k}=m i_{k}$, one obtains that

$$
S_{11}(k \cdot j)=\sqrt{2 \pi} \widetilde{\varphi}_{1}\left(2 \pi m \frac{r}{\omega}\left(i_{k}-i_{j}\right)\right) ;
$$

hence by (2.10) since $m r$ is an integer

$$
S_{11}(k, j)=\left(2 r(1-r)+r^{2}\right) \delta_{j, k} \quad 1 \leq k \leq N, \quad 1 \leq j \leq N,
$$

where $\delta_{j, k}$ is the Kronecker symbol. This shows that $S_{11}=\left(2 r-r^{2}\right) I$. Similarly, by using (3.9) and (3.4), we obtain that $S_{22}=r^{2} I$ and $S_{12}=0$. From (3.3) one finds that the off-diagonal entries of the matrix $S_{21}$ are

$$
S_{21}(k, j)=(1-r) \frac{\omega}{m \pi\left(i_{k}-i_{j}\right)} \quad k \neq j .
$$

The diagonal entries in $S_{21}$ are equal to zero, since $\widetilde{\varphi}_{1}^{\prime}(0)=0$.

In the following theorem we find estimates for the minimum and maximum eigenvalues of the matrix $S_{11}$ and $S_{22}$. The estimates do not depend on the number of missing samples and are simple to compute.

Theorem 4.2. Suppose that $0<r<1$ and $\mathcal{U}=m \mathcal{I}$, where $\mathcal{I}=\left\{i_{1}, i_{2}, \cdots, i_{N}\right\}$ and $m$ is a positive integer. If $m r$ is not integer, then for $j=1, \ldots, N$

$$
\begin{aligned}
& \alpha_{11}<\lambda_{j}\left(S_{11}\right)<\beta_{11} \\
& \alpha_{22}<\lambda_{j}\left(S_{22}\right)<\beta_{22}
\end{aligned}
$$

where

$$
\begin{array}{ll}
\alpha_{11}=\frac{D}{m}\left(1-\frac{D}{4 m}-\frac{1}{4 m}\right), & \beta_{11}=\alpha_{11}+\frac{1}{m} \\
\alpha_{22}=\frac{D}{4 m^{2}}(D-1), & \beta_{22}=\frac{D}{4 m^{2}}(D+1)+\frac{r}{m}
\end{array}
$$

and $D=\lfloor 2 m r\rfloor$. 
Proof. From (2.9), by Fourier inversion and a change of variable, we obtain

$$
\widetilde{\varphi}_{1}(x)=\frac{1}{\sqrt{2 \pi}} \int_{-r}^{r}(1-|y|) e^{i x y \omega / r} d y .
$$

Hence by (3.9) with $l_{k}=m i_{k}$,

$$
S_{11}(k, j)=\int_{-r}^{r}(1-|y|) e^{2 \pi i m y\left(i_{k}-i_{j}\right)} d y .
$$

Let $x=\left(x_{o}, x_{1}, \ldots, x_{N-1}\right)$ be a column-vector in $\mathbb{C}^{N}$ and denote by $x^{*}$ its conjugate transpose. Then

$$
\begin{aligned}
x^{*} S_{11} x=\sum_{k, j=0}^{N-1} \bar{x}_{k} S_{11}(k, j) x_{j} & =\int_{-r}^{r}(1-|y|) \sum_{k, j=0}^{N-1} \bar{x}_{k} x_{j} e^{2 \pi i m y\left(i_{k}-i_{j}\right)} d y \\
& =\int_{-r}^{r}(1-|y|)|P(y)|^{2} d y
\end{aligned}
$$

where $P(y)$ is the $1 / m$-periodic function

$$
P(y)=\sum_{k=0}^{N-1} x_{k} e^{-2 \pi i m y i_{k}} .
$$

By splitting the integral in two parts and changing variable, the above equation may be written

$$
x^{*} S_{11} x=\int_{0}^{r}(1-y) M(y) d y,
$$

where we have set

$$
M(y)=|P(y)|^{2}+|P(-y)|^{2} .
$$

Next we write the integral in (4.11) as a sum of $D+1$ integrals

$$
x^{*} S_{11} x=\sum_{k=0}^{D-1} \int_{k / 2 m}^{(k+1) / 2 m}(1-y) M(y) d y+\int_{D /(2 m)}^{r}(1-y) M(y) d y .
$$

Since $M \geq 0$, we majorize $(1-y)$ in each integral and obtain the estimate

$$
x^{*} S_{11} x \leq \sum_{k=0}^{D-1}\left(1-\frac{k}{2 m}\right) \int_{k / 2 m}^{(k+1) / 2 m} M(y) d y+\left(1-\frac{D}{2 m}\right) \int_{D /(2 m)}^{r} M(y) d y .
$$

Next, we prove that for all $k=0, \ldots, D-1$

$$
\int_{k / 2 m}^{(k+1) / 2 m} M(y) d y=\frac{1}{m} .
$$

Indeed by (4.12), by changing variables and using the $(1 / m)$-periodicity of $P$,

$$
\begin{aligned}
\int_{k / 2 m}^{(k+1) / 2 m} M(y) d y & =\int_{k / 2 m}^{(k+1) / 2 m}|P(y)|^{2} d y+\int_{-(k+1) / 2 m}^{-k / 2 m}|P(y)|^{2} d y \\
& =\int_{k / 2 m}^{(k+1) / 2 m}|P(y)|^{2} d y+\int_{(k+1) / 2 m}^{(k+2) / 2 m}|P(y)|^{2} d y \\
& =\int_{k / 2 m}^{(k+2) / 2 m}|P(y)|^{2} d y=\int_{0}^{1 / m}|P(y)|^{2} d y .
\end{aligned}
$$


By (4.10) and the Plancherel formula, since $x$ has norm equal to 1 , we have

$$
\int_{0}^{1 / m}|P(y)|^{2} d y=\frac{1}{m}
$$

This concludes the proof of equation (4.15). Next we prove that

$$
\int_{D /(2 m)}^{r} M(y) d y<\frac{1}{m}
$$

Indeed, from (4.12), by changing variable in the integral and using the $1 / m$-periodicity of $P$, we obtain

$$
\begin{aligned}
\int_{D /(2 m)}^{r} M(y) d y & =\int_{D /(2 m)}^{r}|P(y)|^{2} d y+\int_{D /(2 m)}^{r}|P(-y)|^{2} d y \\
& =\int_{D /(2 m)}^{r}|P(y)|^{2} d y+\int_{D / m-r}^{D /(2 m)}|P(y)|^{2} d y \\
& =\int_{D / m-r}^{r}|P(y)|^{2} d y .
\end{aligned}
$$

The length $2 r-D / m$ of the interval of integration is less than the period $1 / m$ of $P$, thus inequality (4.17) follows from (4.16).

From (4.14), by using (4.15) and (4.17), we obtain

$$
x^{*} S_{11} x \leq \frac{1}{m}\left[\sum_{k=0}^{D-1}\left(1-\frac{k}{2 m}\right)+\left(1-\frac{D}{2 m}\right)\right]=\frac{D}{m}\left(1-\frac{D}{4 m}-\frac{1}{4 m}\right)+\frac{1}{m}
$$

Thus, we have proved the upper bound for the eigenvalues of $S_{11}$. From (4.13), by observing that the second integral is positive and using (4.15) we obtain

$$
x^{*} S_{11} x \geq \sum_{k=0}^{D-1} \int_{k / 2 m}^{(k+1) / 2 m}(1-y) M(y) d y>\frac{1}{m} \sum_{k=0}^{D-1}\left(1-\frac{k+1}{2 m}\right)=\frac{D}{m}\left(1-\frac{D}{4 m}-\frac{1}{4 m}\right) .
$$

This proves the lower bound and concludes the proof of inequality (4.7). We omit the proof of inequality (4.8), which is similar.

In Table 1 and Table 2, we present some examples: we let $\mathcal{U}=\{0,8,16,24\}$ and compare the minimum and maximum eigenvalues of $S_{11}$ and $S_{22}$ with their estimates given by Theorem 4.2, for various values of $r$.

From Proposition 4.1 one can see that, if $m r$ is an integer, then the eigenvalues of $S_{22}$ are smaller than the eigenvalues of $S_{11}$. The following corollary shows that this is also true when $m r$ is not an integer, provided that $m$ is sufficiently large.

Corollary 4.3. Let $m$ be a positive integer, $\mathcal{U}=m \mathcal{I}$ and $\mathcal{I}=\left\{i_{1}, i_{2}, \cdots, i_{N}\right\}, 0<$ $r<1$. If $m r$ is not an integer and $m>(1+2 r) /(2 r(1-r))$, then

$$
\lambda_{\max }\left(S_{22}\right)<\lambda_{\min }\left(S_{11}\right)
$$




\begin{tabular}{|l|c|c|c|c|}
\hline$r$ & $\alpha_{11}$ & $\lambda_{\min }\left(S_{11}\right)$ & $\lambda_{\max }\left(S_{11}\right)$ & $\beta_{11}$ \\
\hline 0.55 & 0.719 & 0.768 & 0.811 & 0.844 \\
0.6 & 0.773 & 0.813 & 0.859 & 0.898 \\
0.7 & 0.859 & 0.903 & 0.926 & 0.984 \\
0.8 & 0.891 & 0.946 & 0.967 & 1.016 \\
0.9 & 0.929 & 0.984 & 0.998 & 1.055 \\
0.95 & 0.936 & 0.996 & 0.999 & 1.063 \\
\hline
\end{tabular}

Table 1: $\lambda_{\min }\left(S_{11}\right), \lambda_{\max }\left(S_{11}\right)$ and their estimates for $\mathcal{U}=\{0,8,16,24\}$ and several values of $r$.

\begin{tabular}{|l|c|c|c|c|}
\hline$r$ & $\alpha_{22}$ & $\lambda_{\min }\left(S_{22}\right)$ & $\lambda_{\max }\left(S_{22}\right)$ & $\beta_{22}$ \\
\hline 0.55 & 0.219 & 0.271 & 0.315 & 0.350 \\
0.6 & 0.281 & 0.317 & 0.391 & 0.427 \\
0.7 & 0.430 & 0.470 & 0.535 & 0.603 \\
0.8 & 0.516 & 0.594 & 0.659 & 0.709 \\
0.9 & 0.711 & 0.766 & 0.871 & 0.932 \\
0.95 & 0.820 & 0.877 & 0.962 & 1.056 \\
\hline
\end{tabular}

Table 2: $\lambda_{\min }\left(S_{22}\right), \lambda_{\max }\left(S_{22}\right)$ and their estimates for $\mathcal{U}=\{0,8,16,24\}$ and several values of $r$.

Proof. By (4.7) and (4.8) it is sufficient to prove that if $m>(1+2 r) /(2 r(1-r))$, then $\beta_{22}<\alpha_{11}$. This inequality is equivalent to $D^{2} / 2 m+D / 2 m+r-D<0$. Since $2 m r-1<D \leq 2 m r$, we have

$$
\frac{D^{2}}{2 m}+\frac{D}{2 m}+r-D<2 m r(r-1)+2 r+1
$$

from which the corollary follows.

Numerical experiments show that for $m<(1+2 r) /(2 r(1-r))$ the maximum eigenvalue of $S_{22}$ may be larger than the minimum eigenvalue of $S_{11}$.

We shall now describe the behavior of the eigenvalues of the matrix $S$ in dependence of the parameters $r$ and $N$. The numerous experiments that we have performed suggest the conjecture that the eigenvalues of this matrix are real, positive, and less than 1 . In what follows, we have ordered the eigenvalues so that $\lambda_{i}<\lambda_{i+1}$ for $i=0, \ldots, N-2$. Figure 1 shows the largest eigenvalue of $S$ as function of the number $N$ of contiguous points, for various values of $r$. Note that, as $r$ approaches 1 , the largest eigenvalue gets close to 1, i.e. the smallest eigenvalue of the matrix $I-S$ tends to zero. This happens even for a small number of missing samples, as for the one-channel case (see [5], Figure $5)$. Thus, since the spectral condition number of a matrix is greater or equal to the ratio of the absolute values of the largest and the smallest eigenvalues, $\operatorname{cond}(I-S)$ becomes very large. In Table 3 , we show the spectral condition numbers of $(I-S)$ for $\mathcal{U}=\{0,1,2, \cdots, 9\}$, for various values of $r$. 


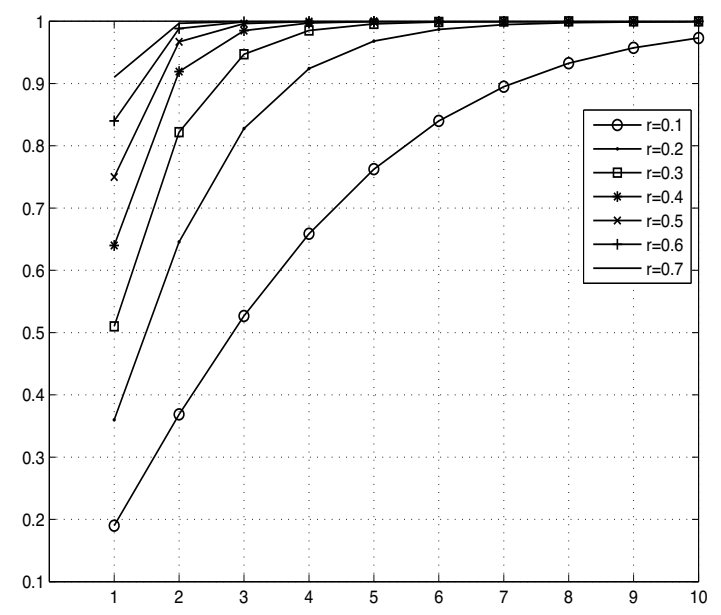

Figure 1: Largest eigenvalues of $S$ as function of $N$ for $\mathcal{I}=\{0,1, \cdots, N-1\}$, for various values of $r$.

\begin{tabular}{|c|c|c|c|}
\hline$r$ & $\operatorname{cond}(I-S)$ & $r$ & $\operatorname{cond}(I-S)$ \\
\hline 0.1 & $8.571 \mathrm{e}+01$ & 0.6 & $2.829 \mathrm{e}+13$ \\
0.2 & $5.870 \mathrm{e}+04$ & 0.7 & $1.661 \mathrm{e}+16$ \\
0.3 & $6.187 \mathrm{e}+05$ & 0.8 & $2.474 \mathrm{e}+17$ \\
0.4 & $1.133 \mathrm{e}+08$ & 0.9 & $4.096 \mathrm{e}+17$ \\
0.5 & $3.513 \mathrm{e}+10$ & & \\
\hline
\end{tabular}

Table 3: Condition number of $I-S$ for $\mathcal{U}=\{0,1,2, \ldots, 9\}$ and $r=0.1,0.2, \ldots, 1$.

Figure 2 shows some of the eigenvalues of $S$, namely $\lambda_{i}, i=1,5,10,11,15,20$, as functions of the parameter $r$ for $\mathcal{I}=\{0,1,2,3, \cdots, 9\}, m=6$ (left) and $m=10$ (right). The dashed lines are the parabolas $2 r-r^{2}$ and $r^{2}$. One can see that, for large values of $m$, the graphs of the eigenvalues concentrate around the graphs of the two parabolas. We observe that, in the one-channel case, the eigenvalues have a similar behavior (see Figure 7 in [5]).

We shall now discuss the behavior of the eigenvalues of the matrix $S$ as the parameter $m$ grows. By using (3.9) with $l_{k}=m i_{k}, k=1, \ldots, N,(2.10),(3.3)$ and (3.4), one can see that, when the parameter $m$ tends to infinity, the off-diagonal entries tend to zero; for each $\epsilon>0$ denote by $m_{\epsilon}$ the positive integer (depending on $N$ ) such that, if $m>m_{\epsilon}$, the off-diagonal entries of the matrix are less than $\epsilon$. For such $m_{\epsilon}$, by the Gershgorin theorem, $N$ eigenvalues lie in the circle of center $2 r-r^{2}$ and radius $\epsilon$ and $N$ eigenvalues lie in the circle of center $r^{2}$ and radius $\epsilon$. Figure 3 illustrates this behavior: it shows the eigenvalues $\lambda_{i} i=1,5,10,11,15,20$ in dependence of the parameter $m$ for $r=.7$ and $\mathcal{I}=\{0,1, \ldots, 9\}$. Note that the limits of the eigenvalues are $2 r-r^{2}$ and $r^{2}$. 

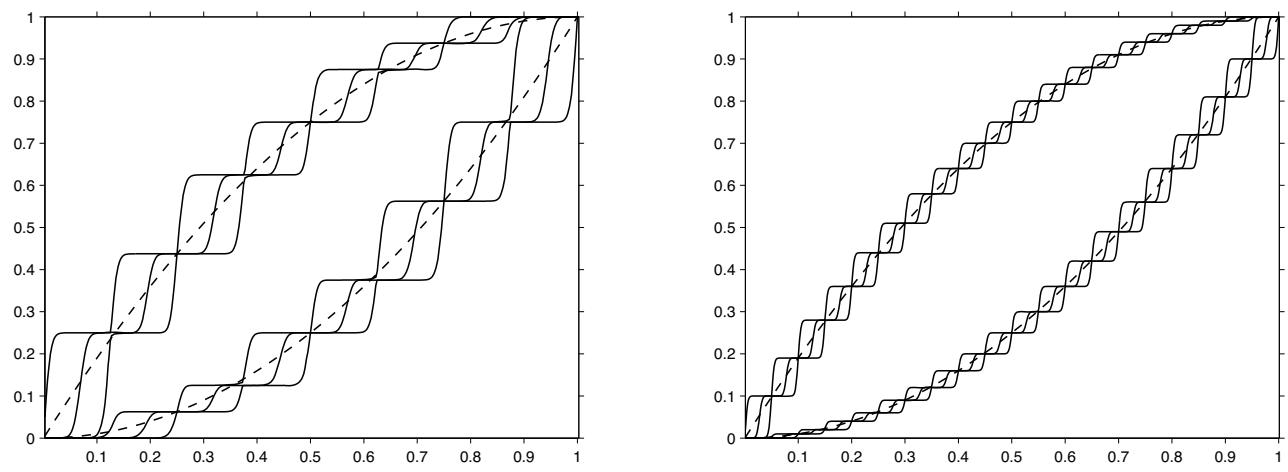

Figure 2: Six eigenvalues of $S$ as functions of $r$ for $\mathcal{U}=\{0,1,2,3, \ldots, 9\}, m=4$ (left) and $m=10$ (right). Dashed lines: parabolas $r^{2}$ and $2 r-r^{2}$

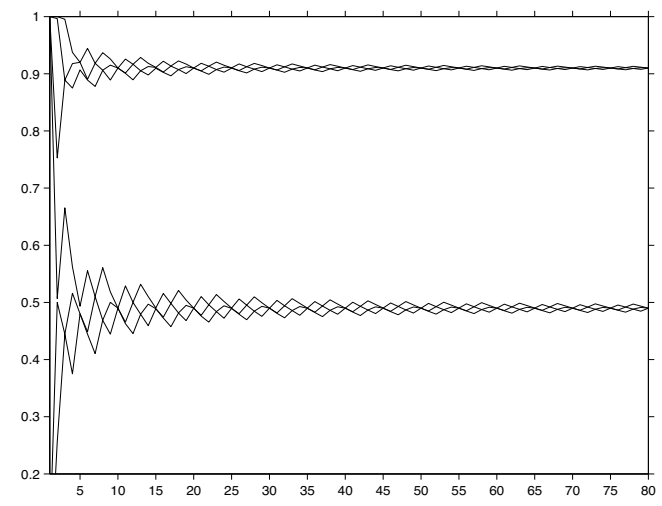

Figure 3: Some of the eigenvalues of $S$ as a function of $m$ for $\mathcal{I}=\{0,1, \ldots, 9\} . r=.7$

\section{Reconstruction: numerical results}

In this section we present some numerical experiments on the recovery of missing samples and on the reconstruction of a signal via oversampling formulas. In particular, we analyze the case of contiguous missing samples, when the problem may become very illconditioned, depending on the parameter $r$ and the number of missing samples. Indeed, if $r$ is close to 1, or the number of missing samples is large, the condition number of the matrices $I-R$ and $I-S$ is large, so that the system greately amplifies the errors in the data. We solve this problem, also in presence of noisy data, by using the Tikhonov regularization technique which we shall now describe briefly. To solve an ill-condizioned linear system $A X=B$ one looks for the solution of the least-square problem

$$
\min _{X}\left(\|A X-B\|^{2}+\lambda\|X\|^{2}\right)
$$

where $\lambda$ is a real positive number, called the regularization parameter. For each value of $\lambda$, this problem has a unique solution $X_{\lambda}$ which is given by

$$
\left(A^{T} A+\lambda I\right) X_{\lambda}=A^{T} B .
$$




\begin{tabular}{|c|r|r|r|r|c|}
\hline \multicolumn{2}{|c|}{} & \multicolumn{2}{|c|}{$M=40$} & \multicolumn{2}{c|}{$M=500$} \\
\hline$x_{k}$ & \multicolumn{1}{|c|}{$g\left(x_{k}\right)$} & \multicolumn{1}{c|}{$g_{k}$} & $e_{k}$ & \multicolumn{1}{c|}{$g_{k}$} & $e_{k}$ \\
\hline 0.0 & 0.1529 & 0.1132 & 0.2598 & 0.1498 & 0.0199 \\
0.6 & -0.2906 & -0.5344 & 0.8390 & -0.3096 & 0.0653 \\
1.2 & 0.0856 & -0.4833 & 6.6498 & 0.0410 & 0.5206 \\
1.8 & 0.9221 & 0.2132 & 0.7687 & 0.8664 & 0.0603 \\
2.4 & 0.8416 & 0.3498 & 0.5844 & 0.8029 & 0.0459 \\
3.0 & 0.0710 & -0.0872 & 2.2288 & 0.0585 & 0.1751 \\
\hline
\end{tabular}

Table 4: Recovered samples with the one-channel formula, $\mathrm{r}=0.6$ : exact samples (col 2), computed samples and relative errors with $M=40$ (col.s 3 and 4 ) and $M=500$ (col.s 5 and $6)$.

If one has an estimate $\epsilon$ of the norm of the error, as in our case, then an approximation of the optimal value of the parameter $\lambda$ can be obtained by using Morozov's discrepancy principle, i.e. by choosing the value of $\lambda$ for which the discrepancy $\left\|A X_{\lambda}-B\right\|^{2}$ is equal to $\epsilon^{2}$ ([1], Section 5).

Following Ferreira, in all our experiments we shall use the test function

$$
g(x)=\operatorname{sinc}(\pi(x-2.1))-0.7 \operatorname{sinc}(\pi(x+1.7))
$$

which has band $[-\pi, \pi][5]$.

First we present some numerical experiments for the recovery of contiguous missing samples via the one-channel formula (4.1). To compute the sum in (4.5), we must truncate it to the values $k$ such that $|k| \leq M$, for some integer $M$, thus introducing an error. In [5] the author makes the choice $M=40$ to reconstruct the signal (5.1) when $\mathcal{U}=\{0,1, \ldots, 5\}$ and $r=0.6$ (hence $t_{o}=0.6$ ). One can verify that for $M=40$ the norm of the truncation error is of order $10^{-3}$ and that the choice $M=500$ reduces it to $10^{-4}$; but a larger value of $M$ does not reduce it further, owing to round-off errors. In Table 4 we show the values $x_{k}=l_{k} t_{o}$ (column 1), the exact samples $g\left(x_{k}\right)$ (column 2), the computed samples $g_{k}$ and the relative errors $e_{k}$ with $M=40$ (columns 3 and 4 ) and with $M=500$ (columns 5 and 6 ). The condition number of the matrix $I-R$ is $3.08 \cdot 10^{4}$. In Figure 4 we have plotted the original and the reconstructed signal in both cases. One can see the better results obtained in the case $M=500$, where we have zoomed the graphs, representing them in the interval $[-5,5]$, to make them distinguishable. By the preceding considerations, in all the experiments described below, we have chosen $M=500$ to reduce the error due to the truncation error.

Next, we have considered the problem of data affected by noise. We have chosen $\mathcal{U}=\{-2,-1,0,1,2,3\}, r=0.6$ and have introduced a random noise of order $10^{-2}$. Note that the condition number of the matrix $I-R$ is still $3.08 \cdot 10^{4}$, since it does not depend on the positions of the contiguous missing samples (see (4.4)). However, since the problem is ill-conditioned, this larger perturbation on the data caused huge errors in the solution of (3.10) (see Table 5, columns 3 and 4). To obtain better results, we have used the Tikhonov regularization technique with the discrepancy principle; we notice that this was possible since the size of the norm of the noise is known. In Table 5 we 

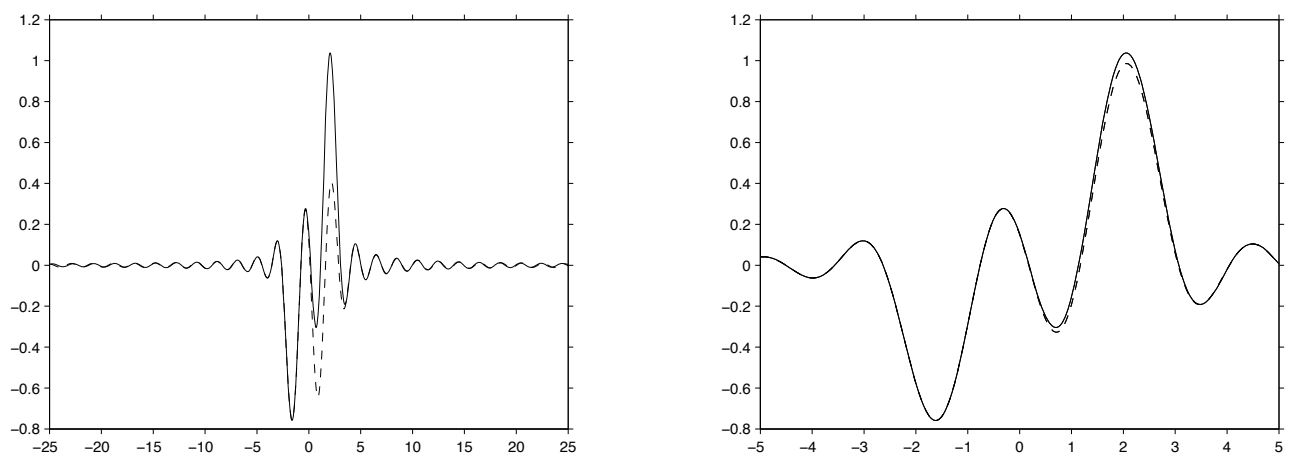

Figure 4: Original (solid line) and reconstructed (dotted line) signal $g, r=.6, \mathcal{U}=$ $\{0,1, \ldots, 5\}$. Truncation $M=40$ (left) and $M=500$ (right), on the interval $[-5,5]$.

show $x_{k}=l_{k} t_{o}$ and the exact values $g\left(x_{k}\right)$ of the missing samples (columns 1 and 2 ), the recovered samples $g_{k}$ and the relative errors $e_{k}$ obtained without regularization (columns 3 and 4 ) and with regularization (columns 5 and 6 ). As in the previous example, by

\begin{tabular}{|r|r|r|r|r|r|}
\hline \multicolumn{2}{|c|}{} & \multicolumn{2}{|c|}{ no regul. } & \multicolumn{2}{c|}{ with regul. } \\
\hline \multicolumn{1}{|c|}{$x_{k}$} & \multicolumn{1}{c|}{$g\left(x_{k}\right)$} & \multicolumn{1}{c|}{$g_{k}$} & \multicolumn{1}{c|}{$e_{k}$} & \multicolumn{1}{c|}{$g_{k}$} & $e_{k}$ \\
\hline-1.2 & -0.5237 & 50.9126 & 98.2227 & -0.4852 & 0.0734 \\
-0.6 & 0.1580 & 180.6426 & 1142.5116 & 0.2141 & 0.3550 \\
0.0 & 0.1529 & 306.9469 & 2006.8097 & 0.1430 & 0.0652 \\
0.6 & -0.2906 & 307.9712 & 1060.8440 & -0.3608 & 0.2416 \\
1.2 & 0.0856 & 183.2893 & 2141.4934 & 0.0435 & 0.4918 \\
1.8 & 0.9221 & 53.8130 & 57.3621 & 0.9248 & 0.0030 \\
\hline
\end{tabular}

Table 5: Recovered samples with the one-channel formula, $\mathrm{r}=0.6$, with noise added: exact samples (col 2), computed samples and relative errors without regularization (col.s 3 and 4 ), and with regularization (col.s 5 and 6).

comparing columns 2 and 5, one can see that the absolute errors are very small: the plots of the real and reconstructed signal would be indistinguishable.

We shall now present some experiments that involve the two-channel formula. To compute the right-hand side of (3.10), we have truncated the sums in (3.6) retaining only the terms for which $|n| \leq 500$. As in the one-channel case, this leads to an error of order $10^{-4}$. Our experiments show that, as in the one-channel case, the recovery and the reconstruction of missing samples of the function and of its derivative is rather efficient if the distance between the missing samples is large and the oversampling parameter $r$ is not too close to 1 . To give an example, for $\mathcal{I}=\{-1,0,1,2,3,4\}, m=4$ and $r=0.7$, the maximum absolute error is less then $8 \cdot 10^{-4}$. The error increases when the parameter $m$ gets smaller, or $r$ larger.

Next, we experiment the recovery of consecutive missing samples of $g$ and $g^{\prime}$ when $\mathcal{U}=\{-2,-1,0,1,2,3\}, r=0.6$ (hence $t_{o}=1.2$ ). The results are shown in Tables 6 


\begin{tabular}{|r|r|r|r|r|c|}
\hline \multicolumn{1}{|c|}{} & \multicolumn{2}{|c|}{ no regul. } & \multicolumn{2}{c|}{ with regul. } \\
\hline \multicolumn{1}{|c|}{$x_{k}$} & \multicolumn{1}{c|}{$g\left(x_{k}\right)$} & \multicolumn{1}{c|}{$g_{k}$} & \multicolumn{1}{c|}{$e_{k}$} & \multicolumn{1}{c|}{$g_{k}$} & $e_{k}$ \\
\hline-2.4 & -0.1868 & -0.0580 & -0.6893 & -0.0785 & 0.5799 \\
-1.2 & -0.5237 & 2.1433 & -5.0929 & -0.4569 & 0.1274 \\
0.0 & 0.1528 & 10.3983 & 67.0174 & 0.1832 & 0.1989 \\
1.2 & 0.0856 & 12.0422 & 139.7624 & -0.1897 & 3.2170 \\
2.4 & 0.8416 & 5.1810 & 5.1561 & -0.1480 & 1.1758 \\
3.6 & -0.1782 & 0.1425 & -1.7997 & -0.4405 & 1.4720 \\
\hline
\end{tabular}

Table 6: Recovered samples of $g$ with two channels, $r=0.6$, no noise added: exact samples (col. 2), computed samples and relative errors (col.s 3 and 4), computed samples and the relative errors (col.s 5 and 6) with regularization.

\begin{tabular}{|r|r|r|r|r|r|}
\hline \multicolumn{1}{|c|}{} & \multicolumn{2}{|c|}{ no regul. } & \multicolumn{2}{c|}{ with regul. } \\
\hline \multicolumn{1}{|c|}{$x_{k}$} & \multicolumn{1}{c|}{$g^{\prime}\left(x_{k}\right)$} & \multicolumn{1}{c|}{$g_{k}^{\prime}$} & \multicolumn{1}{c|}{$e_{k}$} & \multicolumn{1}{c|}{$g_{k}^{\prime}$} & $e_{k}$ \\
\hline-2.4 & -0.9399 & -0.4742 & -0.4955 & -0.7919 & 0.1575 \\
-1.2 & 1.0457 & 5.5797 & 4.3356 & 0.8325 & 0.2039 \\
0.0 & -0.7350 & 5.4046 & -8.3534 & -0.5690 & 0.2258 \\
1.2 & 1.4159 & -2.6352 & 2.8611 & 0.5867 & 0.5857 \\
2.4 & -1.0603 & -7.1265 & -5.7212 & -0.9327 & 0.1203 \\
3.6 & 0.2127 & -0.8092 & 4.8046 & 0.8036 & 2.7786 \\
\hline
\end{tabular}

Table 7: Recovered samples of $g^{\prime}$ with two channels, $r=0.6$, no noise added: exact samples (col. 2), computed samples and relative errors (col.s 3 and 4), computed samples and the relative errors (col.s 5 and 6) with regularization.

and 7. In this case the condition number of the matrix $I-S$ in (3.10) is $3.67 \cdot 10^{7}$, much larger than in the one-channel case. This has the effect of propagating strongly the truncation error, thus producing huge errors of the solution, much worse than in the one-channel case. Thus, with this distribution of missing points and this value of the parameter $r$, the system is very ill-conditioned; an application of the Tikhonov regularization technique with discrepancy principle leads to the results in columns 5 and 6 in Tables 6 and 7. In Figure 5 we show the plots of the original signal $g$ and of the computed one obtained with regularization.

Of course, by using a smaller oversampling parameter, one can reduce the condition number of the matrix $I-S$, obtaining much better results: by using $r=0.3$ (hence $\left.t_{o}=0.6\right)$, and the same set $\mathcal{U}=\{-2,-1,0,1,2,3\}$, one gets cond $(I-S)=1.92 \cdot 10^{4}$. In Section 2 we have observed that, in the two-channel formula, the case $r \in(0,0.5)$ implies oversampling in each channel separately, thus the one-channel formula can be used separately for the signal and its derivative. However, the two-channel formula may be more efficient; we have experimented the recovery of consecutive samples of the function $g$ and its derivative by using the one-channel formula for $g$ and for $g^{\prime}$ (with $r=0.6$ ) and with the two-channel formula (with $r=0.3$ ). Table 8 shows the computed samples $g_{k}$ and the relative errors $e_{k}$, obtained with the one-channel formula (columns 


\begin{tabular}{|r|r|r|r|r|c|}
\hline \multicolumn{1}{|c|}{} & \multicolumn{1}{|c|}{1 chan. } & $r=.6$ & \multicolumn{2}{|c|}{2 chan. } & $r=.3$ \\
\hline \multicolumn{1}{|c|}{$x_{k}$} & \multicolumn{1}{c|}{$g\left(x_{k}\right)$} & \multicolumn{1}{c}{$g_{k}$} & $e_{k}$ & \multicolumn{1}{c|}{$g_{k}$} & $e_{k}$ \\
\hline-1.2 & -0.5237 & -0.5321 & 0.0160 & -0.5261 & 0.0046 \\
-0.6 & 0.1580 & 0.1350 & 0.1454 & 0.1506 & 0.0466 \\
0.0 & 0.1529 & 0.1255 & 0.1790 & 0.1451 & 0.0507 \\
0.6 & -0.2906 & -0.3059 & 0.0527 & -0.2926 & 0.0069 \\
1.2 & 0.0856 & 0.0840 & 0.0184 & 0.0879 & 0.0270 \\
1.8 & 0.9221 & 0.9239 & 0.0020 & 0.9235 & 0.0016 \\
\hline
\end{tabular}

Table 8: Recovered samples of $g$. Col.s 3 and 4: computed samples and relative errors with one channel and $r=0.6$. Col.s 3 and 4: computed samples and relative errors with two channels and $r=0.3$.

3 and 4) and the two-channel formula (columns 5 and 6) for $r=0.6$ and $r=0.3$ respectively. The results for the derivative are even hett.er.

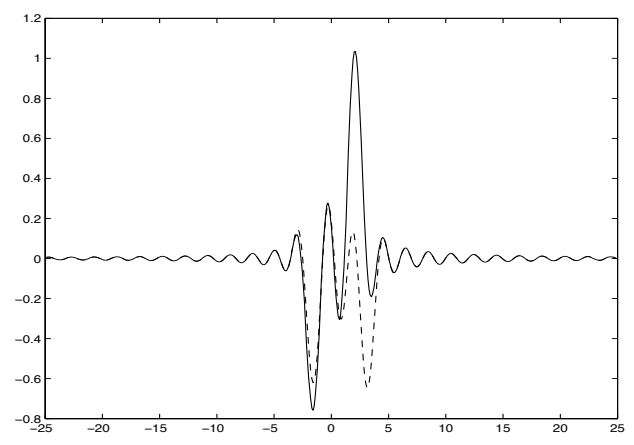

Figure 5: Original and reconstructed signal $g$, samples recovered with regularization, for $\mathcal{U}=\{-2,-1,0,1,2,3\} r=0.6$.

\section{Conclusions}

This paper deals with the recovery of a finite number of missing samples of a bandlimited signal via the two-channel derivative oversampling formula. Unlike the onechannel case, studied in [5], where the reconstruction matrix $R$ is symmetric, the reconstruction matrix $S$ in the two-channel case is no longer symmetric. The matrix $S$ has a $2 \times 2$ block structure, where the diagonal blocks are symmetric and the off-diagonal blocks are antisymmetric. This makes the analysis of the stability of the reconstruction procedure more difficult than in the one-channel case. We analyzed in detail the case where the locations of the missing samples $\mathcal{U}=\left\{m i_{1}, m i_{2}, \ldots, m i_{N}\right\}$ are equally spaced. Here $m$ is a positive integer and the $i_{k}$ 's are consecutive numbers. In this case we have found bounds for the eigenvalues of the diagonal blocks $S_{11}$ and $S_{22}$, depending 
on $m$ and on the oversampling parameter $r$. We proved that, if $m r$ is an integer, then the matrix $S$ is lower triangular and that half of its eigenvalues are equal to $2 r-r^{2}$, while the other half are equal to $r^{2}$. We also presented numerical experiments supporting our conjecture that all the eigenvalues of $S$ are real and lie in $(0,1)$ for every value of $r \in(0,1)$.

In the second part of the paper, we tried the reconstruction procedure on a test function, for a single channel and two channels, both in the case of noise-free and noisy data. In the ill-conditioned problem of consecutive missing samples we have found a regularized solution via the Tikhonov method with the discrepancy principle.

\section{References}

[1] M. Bertero and P. Boccacci, Introduction to Inverse Problems in Imaging, Institute of Physics Publ., Bristol, 1998.

[2] V. Del Prete, Recovery of missing samples in oversampling formulas for bandlimited functions, Sampl. Theory Signal Image Process., 8(2), 161 - 180, 2009.

[3] V. Del Prete, Frames and oversampling formulas for band-limited functions, Ann. Mat. Pura Appl. (4), 189(3), 445-474, 2010.

[4] P.J.S.G. Ferreira, Incomplete sampling series and the recovery of missing samples from oversampled band-limited signals, IEEE Trans. Signal Processing, 40(1), 225 - 227, 1992.

[5] P.J.S.G. Ferreira, The stability of a procedure for the recovery of lost samples in band-limited signals, Signal Processing, 40(3), 195-205, 1994.

[6] P.J.S.G. Ferreira, Stability issues in error control coding in the complex field, interpolation and frame bounds, IEEE Sig. Proc. Letters, 7(3), 57-59, 2000.

[7] J.R. Higgins, Sampling Theory in Fourier and Signal Analysis. Foundations, Oxford University Press, Oxford, 1996.

[8] J. M. Kim and K. H. Kwon, Recovery of finite missing samples in two-channel oversampling, Sampl. Theory Signal Image Process., 6(2), 185 - 198, 2007.

[9] V.A. Morozov, Methods for Solving Incorrectly Posed Problems, Springer, Berlin, 1984.

[10] R.J. Marks II, Restoring lost samples from an oversampled band-limited signal, Proceedings of the IEEE International Conference on Acoustics, Speech, and Signal Processing, ICASSP-31, 3, 752 - 755, June 1983.

[11] R.J. Marks II, Introduction to Shannon Sampling and Interpolation Theory, Springer, Berlin, 1991.

[12] D.M.S. Santos and P.J.S.G. Ferreira, Reconstruction from missing function and derivative samples and oversampled filter banks, Proceedings of the IEEE International Conference on Acoustics, Speech, and Signal Processing, ICASSP 04, 3, 2004, 941 - 944. 
[13] D.M.S. Santos, P.J.S.G. Ferreira and J.M.N. Vieira, Study of the recovery of missing samples for function and derivative oversampled filter banks, Proceedings of the 2005 International Workshop on Sampling Theory and Applications, SampTA 2005, Samsun, Turkey. 